\title{
Estimation of Pump-Curves using Genetic Algorithms
}

\author{
Gerulf K.M. Pedersen \\ Department of Cognitive Psychology \\ University of Würzburg \\ 97070 Würzburg, Germany \\ gerulf@psychologie.uni-wuerzburg.de
}

\author{
Zhenyu Yang \\ Esbjerg Institute of Technology \\ Aalborg University \\ Esbjerg, Denmark \\ yang@cs.aaue.dk
}

\begin{abstract}
This paper presents a variety of different ways of estimating the general parameters for pump-curves. First a formulation is made that converts the problem into estimating four parameters in this general formulation. Then three different methods that utilize the general formulation are presented. The estimated parameters for each method are used in generating the desired pump-curves, and the quality of the estimates are determined. The paper finishes with a recommendation that, for estimation of the generalized parameters, the method utilizing speed differences is preferable over the other methods discussed in the paper.
\end{abstract}

\section{Categories and Subject Descriptors}

I.6.5 [Computing Methodologies]: Model Development; J.6 [Computer Applications]: Computer-aided engineering-Computer-aided design

\section{General Terms}

Experimentation, Verification

\section{Keywords}

Pump curves, Parameter estimation, Curve fitting, Simple Genetic Algorithm

\section{INTRODUCTION}

Today one of the most dominant electrical applications worldwide is the electrical pump[5]. From the most modern parts of the world to the most primitive parts it can be seen that the pump has found its use in a variety of application areas, including water supply, waste treatment, and hydraulics. One of the key elements when using pumps for different applications is that the right kind of pump is chosen for a given application. This will ensure that the pump will perform as desired.

When choosing a pump for an application it is also necessary to take other issues into account than just the type

Permission to make digital or hard copies of all or part of this work for personal or classroom use is granted without fee provided that copies are not made or distributed for profit or commercial advantage and that copies bear this notice and the full citation on the first page. To copy otherwise, to republish, to post on servers or to redistribute to lists, requires prior specific permission and/or a fee.

GECCO'08, July 12-16, 2008, Atlanta, Georgia, USA.

Copyright 2008 ACM 978-1-60558-130-9/08/07...\$5.00. of pump. Besides considering the environment in which the pump will be operating, the issue of choosing the right size of pump is important. Each pump has a range where it is most efficient. In order to ensure the most efficient operation, the typical duty point of the pump should be in that range for as much of the time as possible. It would otherwise lead to unnecessary energy losses [5]. One way to compare different pumps is to look at the pump-curves and choosing the pump that best matches the specific need. It is definitely not an issue of just choosing a pump that is large enough; it should be suitable.

The way pump-curves are usually generated for a pump is for a set of pressure and flow measurements to be made for different system loads at one specific speed, usually in the range of $85 \%-100 \%$ of the maximally rated speed. Once this set of measurements has been made, the performance of the pump at other speeds are calculated using certain affinity laws[5], which approximate the pump performance at other speeds. However, it remains to be seen at which speed it might be best to perform these measurements before applying the affinity laws. Another interesting thing worth investigating is the approximation error that occurs when the affinity laws are applied.

This paper will try several different methods for estimating the best possible base pump-curve for a given pump using a simple genetic algorithm. Estimation of the pumpcurve could also have been attempted using a nonlinear version of the conjugate gradient method $[2,1]$ or a LevenbergMarquardt method [6] but a genetic algorithm was chosen since it is capable of escaping the local extrema that is expected for this particular problem.

Once a base pump-curve has been found it can also be checked whether the precision of the affinity laws are adequate for various design purposes. So, a side bonus of this paper is an investigation into the validity of the widely used affinity laws.

First, in Section 2 a description of the pump is given, both physically as well as mathematically. Then, in Section 3 the different methods for estimating the base pump-curve is given. Section 4 explains how the simple genetic algorithm is implemented and used for the estimation of the base pumpcurves. The results follow in section 5 and the conclusion wraps it all up in section 6.

\section{PUMP DESCRIPTION}

This section will give a description of the pump chosen for this investigation. First, a physical description of the pump will be given followed by a mathematical description. 


\subsection{Physical System}

The pump used for the investigation of this paper is a Grundfos CRE5-8 centrifugal pump as shown in Figure 1 which was generously supplied by Grundfos for this and other investigations [7].

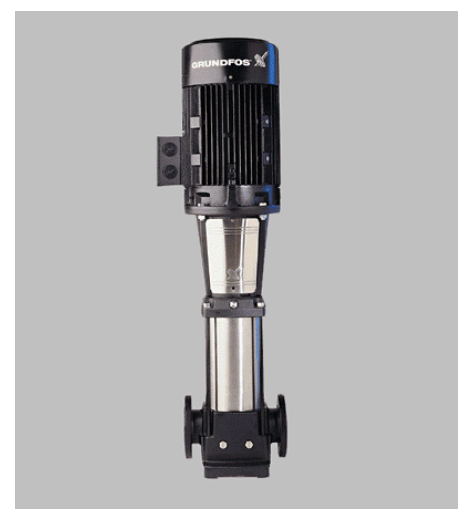

Figure 1: A centrifugal pump.

It is a variable speed pump, whose speed is controlled by a low voltage DC input signal. The DC signal goes into a frequency converter which transforms the signal into a varying AC signal that powers an induction motor. The induction motor is connected to the centrifugal pump through the shaft.

For the specific pump a measurement of the pump speed cannot be performed automatically as the model does not come with a built-in tachometer/encoder. In order to avoid making such speed measurements manually, the input signal to the pump, a DC voltage in the range $0-10 \mathrm{~V}$, is used to represent the speed in the remainder of this paper. This might cause some approximation errors, especially for high pressure conditions, as the frequency converter and induction motor might cause the actual speed of the pump to not have a linear dependency of the reference speed. However, it is deemed a valid approximation, since the measurements are only made during static periods, meaning that the speed remains unchanged.

\subsection{Mathematical Model}

Finding the coefficients for the mathematical model of the pump is the goal of this paper, but before the coefficients can be found it is necessary to take a look at the structure of the model. Once the structure has been found the different methods for finding the coefficients will be presented. The model that will be given here is purely static and does not take the dynamics of any set point changes into account.

Normally, when discussing pumps, the key features that are discussed are flow and pressure. However, when comparing pumps it is more common to consider the flow and the head. The head is given by

$$
H=\frac{\Delta p}{g \rho},
$$

where $g$ is the gravity constant, $\rho$ is the density of the liquid being pumped and $\Delta p$ is the pressure difference generated by the pump. The reason for this transformation is to make the comparison independent of the medium being pumped and thus also eliminate any effect of temperature variances of the medium. The head basically states to which maximum height any liquid can be pumped for that given setting of the pump.

The mathematical model of a pump is usually described using a second-, third-, or fourth-order polynomial relationship between flow $Q$ and head $H$. This is partly due to the friction losses which are quadratic with regard to the flow. These are dominant for high flow rates. Similarly, at low flow rates leakage flows are dominant and has a low order polynomial impact on the pump performance. In this paper a second order approximation to the centrifugal pump will be used and it is also the coefficients for that model that will be estimated later on. The second order model is chosen as it is easy to relate it to the physical properties of the performance of the pump.

Using a second order polynomial, the relationship between flow and head can be written as

$$
H(\omega)=a(\omega) Q(\omega)^{2}+b(\omega) Q(\omega)+c(\omega),
$$

where $\omega$ is the angular velocity of the pump and the coefficients $a(\omega), b(\omega)$, and $c(\omega)$ are speed dependent coefficients.

How the coefficients depend on the speed can be quite complex. However, if the losses are neglected then a set of affinity laws can be used to approximate $a(\omega), b(\omega)$, and $c(\omega)$.

The affinity laws for a system with a fixed load are given as

$$
\begin{aligned}
Q\left(\omega_{B}\right) & =\frac{\omega_{B}}{\omega_{A}} Q\left(\omega_{A}\right) \\
H\left(\omega_{B}\right) & =\left(\frac{\omega_{B}}{\omega_{A}}\right)^{2} H\left(\omega_{A}\right),
\end{aligned}
$$

where $\omega_{A}$ and $\omega_{B}$ are specific speeds of the pump. Introduction of a baseline speed $\omega_{0}$, from which the pump curve will be calculated using affinity laws, results in the following relationship between head and flow

$$
H(\omega)=a_{0} Q(\omega)^{2}+b_{0} \frac{\omega}{\omega_{0}} Q(\omega)+c_{0}\left(\frac{\omega}{\omega_{0}}\right)^{2},
$$

which will be referred to as the general pump curve. Comparing this to equation (2) yields

$$
\begin{aligned}
a(\omega) & =a_{0} \\
b(\omega) & =b_{0} \frac{\omega}{\omega_{0}} \\
c(\omega) & =c_{0} \frac{\omega^{2}}{\omega_{0}^{2}} .
\end{aligned}
$$

It is thus evident that estimation of the four parameters $a_{0}, b_{0}, c_{0}$, and $\omega_{0}$ should result in an approximate mathematical model of a centrifugal pump.

Based on the findings so far it is just a matter of finding the parameters $a_{0}, b_{0}, c_{0}$, and $\omega_{0}$. In the following section three different methods for estimating these parameters will be given.

\section{ESTIMATION OF PUMP-CURVE PARAM- ETERS}

The authors are not aware of any previous methods for estimating pump-curves based on measurements at multiple speeds. The methods proposed in this section are based on different properties of the affinity laws for obtaining the best suitable parameters for the model. 
All of the methods used for estimating the general pumpcurve parameters are based on some assumptions. It is assumed that the parameters are bounded and that $a_{0}$ and $b_{0}$ are negative and $c_{0}$ and $\omega_{0}$ are positive.

The assumption for $a_{0}$ is obvious as it would otherwise be possible to obtain infinite head and infinite flow. $b_{0}$ is negative because the peak of the second order polynomial should lie in the left half plane. This ensures a monotonically decreasing relationship between the head and flow in the operating range, with positive flow and positive head only. $c_{0}$ must be positive because $a_{0}$ is negative. If not then the pump curve would not enter the operating range that was just specified. Finally, $\omega_{0}$ must be positive due to the limitation that the pump cannot run backwards.

With the limitations of the parameters in place it is time to look at the different methods. One method is based on minimization of the least squares error using Euclidean distance of each measurement to the pump-curve. Another method minimizes the least squares error of the speed difference between the estimated pump curve and the measurement. The third method minimizes the least squares error between an estimate of the hydraulic power and the actual hydraulic power obtained through a measurement.

First, the method that minimizes the least squares error of the Euclidean distance will be presented.

\subsection{Euclidean Distance Based Estimation}

The Euclidean distance based estimation method is more or less straightforward.

For different speeds and different system loads a series of measurements will be made. Each of these measurements contains information of the pressure, the flow and the speed reference to the pump. For each of these measurements the speed reference sent to the pump will be used to estimate the pump curve based on the $a_{0}, b_{0}, c_{0}$, and $\omega_{0}$ parameters. It is then just a matter of calculating the Euclidean distance between the estimated pump-curve and the actual measurement.

Each measurement consists of the 3 -tuple $(\Delta \hat{p}, \hat{Q}, \hat{\omega})$ where it should be remembered that $\hat{\omega}$ is the reference given to the system and not a measurement of the actual speed. For compatibility with the equations so far the pressure difference, $\Delta \hat{p}$, should be converted into a measured head, $\hat{H}$, using equation (1).

An estimate of the pump curve can then be given by insertion of $\hat{\omega}$ into equation (5)

$$
\tilde{H}=a_{0} \tilde{Q}^{2}+b_{0} \frac{\hat{\omega}}{\omega_{0}} \tilde{Q}+c_{0}\left(\frac{\hat{\omega}}{\omega_{0}}\right)^{2},
$$

which is a second order polynomial with regard to the estimated head and flow, $\tilde{H}$ and $\tilde{Q}$.

The square of the Euclidean distance between the estimated pump-curve and the measurement, $d^{2}$, can be expressed as a fourth order polynomial

$$
\begin{aligned}
d^{2}= & (\tilde{Q}-\hat{Q})^{2}+(\tilde{H}-\hat{H})^{2} \\
= & \tilde{Q}^{2}+\hat{Q}^{2}-2 \tilde{Q} \hat{Q}+\left(a_{0} \tilde{Q}^{2}+b_{0} \frac{\hat{\omega}}{\omega_{0}} \tilde{Q}+c_{0}\left(\frac{\hat{\omega}}{\omega_{0}}\right)^{2}\right)^{2} \\
& +\hat{H}^{2}-2\left(a_{0} \tilde{Q}^{2}+b_{0} \frac{\hat{\omega}}{\omega_{0}} \tilde{Q}+c_{0}\left(\frac{\hat{\omega}}{\omega_{0}}\right)^{2}\right) \hat{H}
\end{aligned}
$$

The minimal distance can be found by taking the derivative with regard to $\tilde{Q}$, setting the derivative equal to zero, solving it, and choosing the purely real value that inserted into equation (10) results in the smallest distance. Detailed calculations are not given here due to limited space.

Some care should be exerted when solving the third order polynomial resulting from taking the derivative. It will always have at least one purely real value, but the remaining two values can be either purely real or complex. If they are complex the single real value is the desired one and the distance can be calculated using that one. If all the values are real, then the one resulting in the smallest distance when inserted into (10) should be used. The distances are then summed for each measurement resulting in the total error of the estimation.

This method is quite straightforward and calculates the minimal Euclidean distance precisely. However, the drawback is that it is necessary to solve a third order polynomial for each measurement point which can result in a heavy computational load when running the genetic algorithm.

The next method is based on speed difference.

\subsection{Speed Difference Based Estimation}

The speed difference estimation method is more or less the opposite of the Euclidean method described previously. Instead of calculating the estimated pump-curve based on the speed reference, the measurement of the head and flow are used in connection with the model to calculate an estimate of the speed. The difference between the estimate of the speed and the actual speed reference can then be found for all measurements, summed and used in the genetic algorithm.

The basis for the calculation is the measurement of the head and flow, $(\hat{Q}, \hat{H})$, inserted into equation (5)

$$
\hat{H}=a_{0} \hat{Q}^{2}+b_{0} \frac{\hat{Q}}{\omega_{0}} \tilde{\omega}+c_{0}\left(\frac{1}{\omega_{0}}\right)^{2} \tilde{\omega}^{2},
$$

which is a second order polynomial with regard to the estimated speed $\tilde{\omega}$. The restriction of the parameters $a_{0}, b_{0}, c_{0}$, and $\omega_{0}$ as well as the fact that the measured head $\hat{H}$ and the measured flow $\hat{Q}$ are positive guarantees the existence of two non-complex solutions to the polynomial. In fact, one of the solutions will always be positive and the other one will be negative.

It is then just a matter of solving the polynomial with regard to $\tilde{\omega}$, choosing the positive solution that results, and taking the absolute value of the difference between that solution and the actual speed reference

$$
\omega_{\text {dif }}=|\tilde{\omega}-\hat{\omega}| .
$$

This can be done for each measurement and summation of these then results in a performance measure that can be used in the simple genetic algorithm to obtain the best possible estimates of $a_{0}, b_{0}, c_{0}$, and $\omega_{0}$. An advantage to this method compared to the previously suggested method is that it only requires that a second order polynomial is solved for each measurement.

It is now time to take a look at the third method proposed in this paper for estimating the pump-curve parameters.

\subsection{Hydraulic Power Based Estimation}

The third method for finding the pump-curve parameters are based on an expression for the hydraulic power delivered 
to the liquid by the pump. The hydraulic power is dependent on both the head and the flow and it is thus possible to use measurements of those variables to correct the estimated parameters of the pump-curve.

The hydraulic power is given by

$$
P_{h y d}(\omega)=H(\omega) g \rho Q(\omega),
$$

and by inserting the measurements of flow $\hat{Q}$ and head $\hat{H}$ into that, the measured hydraulic power $\hat{P}_{h y d}$ can be found. This has to be compared to the estimated hydraulic power based on the pump-curve.

Using the base pump-curve formula in equation (5) it is also possible to get an expression for the hydraulic power in terms of the parameters $a_{0}, b_{0}, c_{0}$, and $\omega_{0}$,

$$
\tilde{P}_{h y d}=g \rho\left(a_{0} \tilde{Q}^{3}+b_{0} \frac{\omega}{\omega_{0}} \tilde{Q}^{2}+c_{0}\left(\frac{\omega}{\omega_{0}}\right)^{2} \tilde{Q}\right) .
$$

Once $\tilde{P}_{h y d}$ has been determined it is simple to calculate the difference in hydraulic power using

$$
P_{h y d_{\text {diff }}}=\left|\tilde{P}_{h y d}-\hat{P}_{h y d}\right|,
$$

and summing over all the available measurements.

The one thing to notice when determining $\tilde{P}_{h y d}$ is that a value for $\tilde{Q}$ is needed. This flow-value can be determined in a number of ways. One way is to simply use the measured value $\hat{Q}$ directly. Another way is to calculate $\tilde{Q}$ as the point on the pump-curve that is closest to the measurement, similarly to the first estimation method using Euclidean distances. Both of these ways of determining $\tilde{Q}$ will be tested in the algorithm.

Before the genetic algorithm can be used to solve the problem presented through the proposed methods it is first necessary to decide on the various parameters required to obtain usable results.

\section{SIMPLE GENETIC ALGORITHM}

This section describes the genetic algorithm and the corresponding run-time parameters that were used for finding the best estimate of the pump-curve parameters.

It was decided to use a simple real valued genetic algorithm for the optimization of the parameters. No special requirements were set, except for an easy-to-use interface through Matlab. Many different algorithms live up to this criteria, but in order to keep the focus on the estimation of the parameters for the given problem it was decided to go with the simplest possible implementation.

The choice fell on a genetic algorithm implementation done by Kumara Sastry [8], which provides a variety of different options. One of the benefits for this implementation is that it includes formulas for calculating default values of different parameters, such as population size and maximum number of generations based. These default values are quite good and based on various genetic algorithm analysis performed previously [4, 9].

The run-time parameters for all the experiments performed in this paper are summarized in Table 1 .

The population size of 172 is the default value, which seemed appropriate for this investigation. The population size is small enough to not cause an excessive amount of computation and should be large enough to prevent premature convergence and maintain diversity. The maximum
Table 1: Parameters used for running the simple genetic algorithm.

\begin{tabular}{|c|c|}
\hline Parameter & Setting \\
\hline \hline Population size & 172 \\
\hline Maximum no. of generations & 149 \\
\hline No. of runs & 30 \\
\hline Representation type & Real values \\
\hline Selection strategy & Tournament \\
\hline Tournament size & 2 \\
\hline Crossover type & SBX \\
\hline Crossover probability & 0.9 \\
\hline SBX parameter & 10 \\
\hline Mutation type & Polynomial \\
\hline Mutation probability & 0.1 \\
\hline Polynomial parameter & 20 \\
\hline
\end{tabular}

number of generations of 149 is a considerable increase of the default value of 28 . To begin with this default value was thought of as being somewhat low, and some preliminary test showed that the algorithm could not really converge within that time span. Using trial and error the maximum number of generations were set at 149 as it seemed that the algorithm usually had converged at that point. It was, however, also decided to run the algorithm 30 times to ensure that the algorithm obtained statistically significant results. The best fit would then be determined as the best individual obtained among all 30 runs.

A real valued representation was chosen arbitrarily. It is expected that a binary representation will produce similar results to those obtained in this paper. An individual will consist of the four parameters, $a_{0}, b_{0}, c_{0}$, and $\omega_{0}$. A range for the parameters was determined based on the previous assumptions and are summarized in Table 2.

Table 2: Parameter ranges.

\begin{tabular}{|c|c|}
\hline Parameter & Range \\
\hline \hline$a_{0}$ & {$[-10,0]$} \\
\hline$b_{0}$ & {$[-10,0]$} \\
\hline$c_{0}$ & {$[0,10]$} \\
\hline$\omega_{0}$ & {$[0,10]$} \\
\hline
\end{tabular}

It should here be noted that the ranges for $a_{0}, b_{0}, c_{0}$, and $\omega_{0}$ are not based on the equations using head, but has been converted back into equations using pressure. This makes it easier to compare the results directly with the pressure measurements of the physical system which used water as the medium being pumped.

The selection strategy chosen is tournament selection which is a default value as well as the tournament size of 2 . The crossover type, probability and SBX parameter ([3]) were also all chosen to their default values, which were deemed adequate. Similarly for mutation, the type, probability, and polynomial parameter were chosen to their default values.

With these preliminary details in place, the more specific implementation details, that needed to be handled before any result could be obtained, will be presented.

\subsection{Implementation}

Before the estimation methods can be tested it is necessary to make some measurements of the pump in question. 
So, a series of measurements was made while the pump was subjected to different speed references and different system loads. A total of 21 different reference speeds were combined with 18 different system loads for a total of 378 measurements. For each measurement the speed reference, pressure, flow, and power consumption were recorded. The power was measured due to a desire to use the results of this paper for further optimization purposes. However, the power measurements also indicated that the pump does not necessarily follow the commanded reference input in the interval $0-10 \mathrm{~V}$, which corresponds linearly to a pump speed reference of $0-100 \%$. This can be seen from Figure 2 .

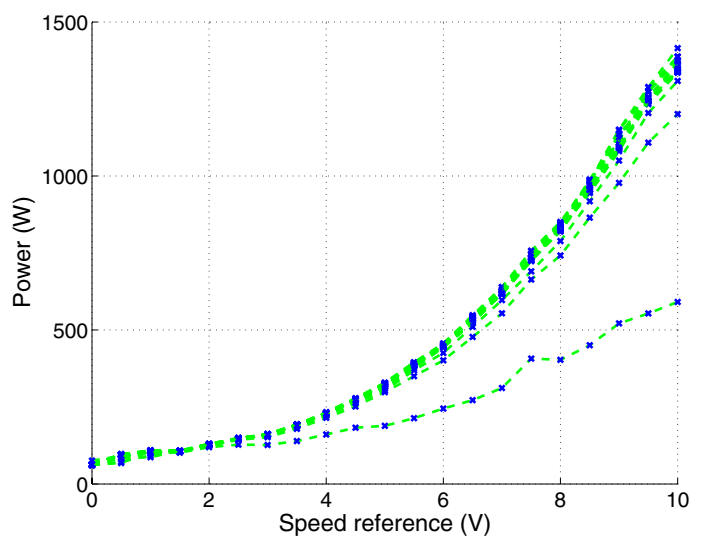

Figure 2: Power consumption of pump for different system curves.

The figure shows that for certain system loads the pump does not consume as much power as for most of the other situations. It has been investigated that this happens for high system loads, which occur when the valve in the system is nearly or fully closed. It is assumed that this reduction in power might be caused by the controller of the induction motor implemented in the frequency converter. The load simply becomes so high that the controller reduces the speed below the commanded reference.

This phenomenon will possibly affect the results of the estimation methods and the extent of the influence will be discussed after the results have been obtained.

Once these measurements had been obtained it was possible to run the proposed estimation algorithms. The results of the algorithms will be presented next.

\section{RESULTS}

The results will be presented in the same order as the methods were described. The first result to be presented is thus the method based on Euclidean distances.

\subsection{Results for Euclidean Distances}

The estimation algorithm was run 30 times and the evolution of the best individual for each of those runs is plotted in Figure 3.

In the figure it is seen that there is a clear tendency for the best individual to have a relatively low value for the base speed, $\omega_{0}$ (cyan), in the range 0 to 2 . There are, however, also a large number of runs where the base speed have a high value around 10, but this is somewhat obscured by the values of $c_{0}$ (red). It is also quite evident that the zeroth

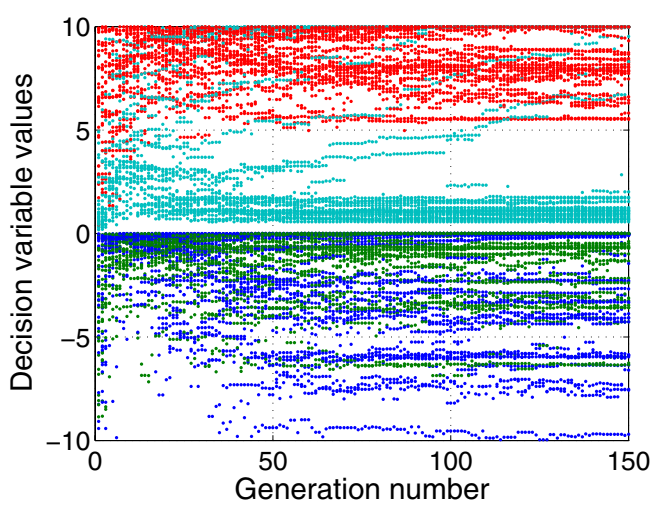

Figure 3: Distribution of pump-curve parameters during evolution using Euclidean distance $\left(a_{0}\right.$ : blue, $b_{0}$ : green, $c_{0}:$ red, $\omega_{0}:$ cyan $)$.

order term, $c_{0}$, tends to be in the upper part of the range $[0,10]$.

The reason why there is such a large spread of the value for $c_{0}$ could be due to the fact that the intersection with the head/pressure-axis is both sensitive to $c_{0}$ as well as the value of the base speed $\omega_{0}$ for the pump-curve, see equation (5).

The values for $b_{0}$ (green), which determines the top-point of the pump-curve, are obscured by the many different values for $a_{0}$ (blue). The values for $b_{0}$ are mostly in the top of the $[-10,0]$ range, indicating that the top-point of the pumpcurve should be located close to the head/pressure-axis.

The values for $a_{0}$ (blue), which determines the steepness of the pump curve, fluctuates a lot in the entire $[-10,0]$ range. However, the majority of runs tends to evolve towards high values.

The best individual among the runs have the values: $\mathbf{a}_{0}=-0.03, b_{0}=-0.08, c_{0}=5.64$, and $\omega_{0}=\mathbf{1 0 . 0 0}$.

An interesting observation is that the 12 runs where the best individual had a base speed of 9 or higher were also the runs where the best individuals had the lowest fitness values compared to the other 18 runs. This indicates that the fitness function had one or more local minima where the algorithm had a good chance of getting stuck.

The best set of parameter values was used to generate the pump-curves seen in Figure 4.

The figure shows that the pump-curves match the measurements quite well. There is of course a bias towards more precise pump-curves for the higher speeds as the affinity laws make the lower speed estimates less precise since they are furthest away from the base curve.

\subsection{Results for Speed Differences}

The evolution of the estimated parameters using speed differences are plotted in Figure 5.

The figure shows that the solutions evolve quite similarly to the Euclidean distances case. The values for $a_{0}$ are in this case more located towards the top of the $[-10,0]$ range, whereas the values for $b_{0}$ in some runs have a tendency of becoming a little lower. The values for $c_{0}$ are again in the upper part of the $[0,10]$ range, and the base speed $\omega_{0}$ has a little more spread. The main part of the runs indicate that low values of $\omega_{0}$ are better. 


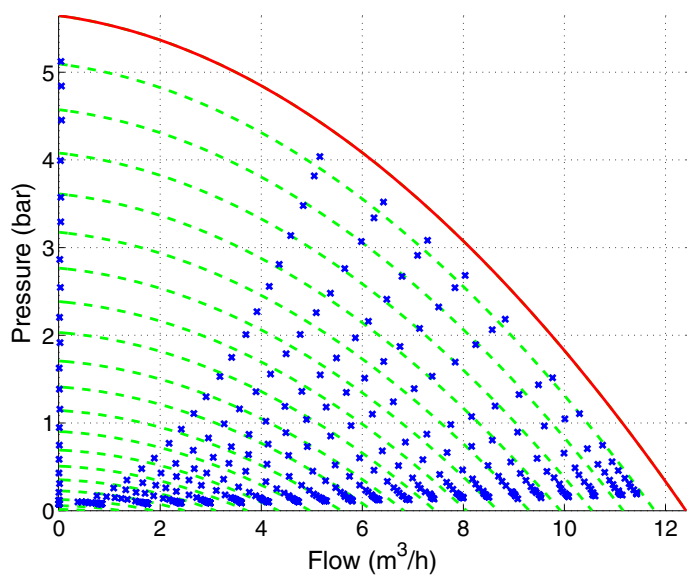

Figure 4: Best estimated pump-curves using Euclidean distance (base curve: solid red, estimated curves: dashed green, measurements: blue points).

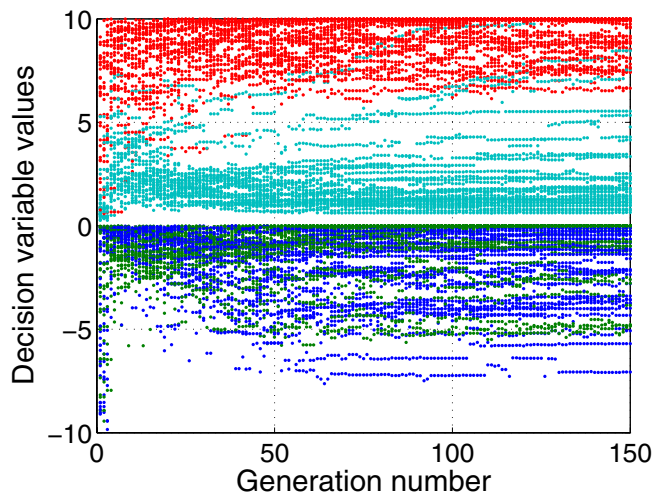

Figure 5: Distribution of pump-curve parameters during evolution using speed difference $\left(a_{0}\right.$ : blue, $b_{0}$ : green, $c_{0}$ : red, $\omega_{0}$ : cyan).

The best individual is obtained for the parameter values: $a_{0}=-0.05, b_{0}=-0.03, c_{0}=0.67$, and $\omega_{0}=2.71$.

In this case there is no clear tendency towards specific values of either parameters. The best individual of the second best run prefers a high value of $\omega_{0}$, which is contradictory to the best overall individual which has a low $\omega_{0}$ value.

The pump-curves obtained using the best set of parameters for the speed difference method are plotted in figure 6.

It can be seen that the base speed is quite low. This results in the pump-curves estimated for high speeds not matching the measurements too well. Further, for high speeds, the shape of the pump curve near the flow-axis does not match the measurements too well either.

\subsection{Results for Hydraulic Power}

The last set of results come from the method based on the formula for hydraulic power given in equation (15). First, the evolution of the parameters where $\tilde{Q}$ is determined by the smallest Euclidean distance are shown in figure 10.

It is very clear that the parameters $a_{0}$ and $b_{0}$ both converge to the lowest possible value. Also, the base speed $\omega_{0}$

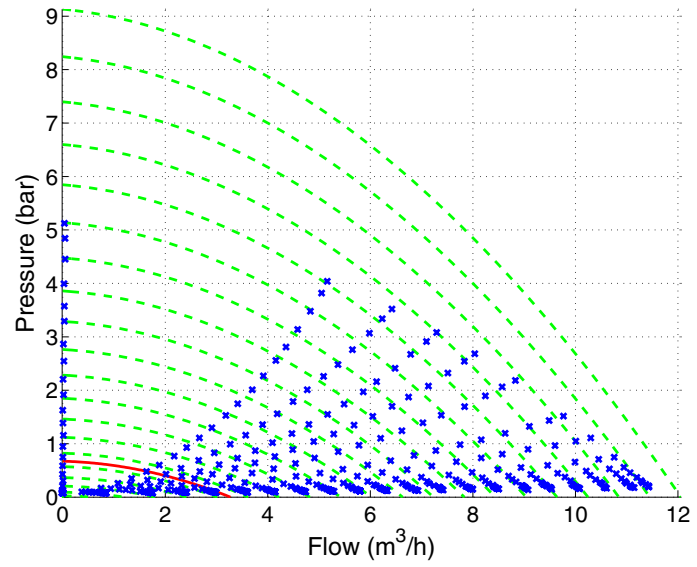

Figure 6: Best estimated pump-curves using speed difference (base curve: solid red, estimated curves: dashed green, measurements: blue points).

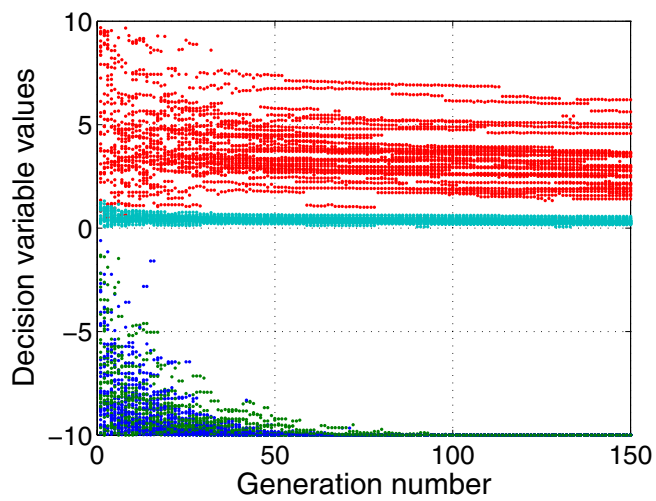

Figure 7: Distribution of pump-curve parameters during evolution using hydraulic power with flow based on minimum Euclidean distance $\left(a_{0}\right.$ : blue, $b_{0}$ : green, $c_{0}:$ red, $\omega_{0}:$ cyan).

converges to low values, even though it never reaches the lower boundary of zero. Finally it can be seen that the final parameter $c_{0}$ tends to settle in the lower part of the $[0,10]$ range.

The best set of parameters for this method is:

$\mathbf{a}_{0}=-10.00, \mathbf{b}_{0}=-10.00, \mathbf{c}_{0}=0.51$, and $\omega_{0}=0.07$.

It is seen that the best set of parameters actually have a $c_{0}$ value which is lower than most of the other runs when using this method. It is, however, possible that very low values of $c_{0}$ are obscured by the dense coverage of $\omega_{0}$ in that area.

The pump-curves obtained using the best set of parameters are shown in figure 8.

The pump-curves are clearly not good approximations when compared to the measurements on which they are based. The maximum values are simply too high. An additional plot of the pump curves was made such that the measurements can be better compared to the curves. This plot is shown in Figure 9.

In the figure it can be seen that the pump curves are far from most of the measurements. Additionally, it can be 


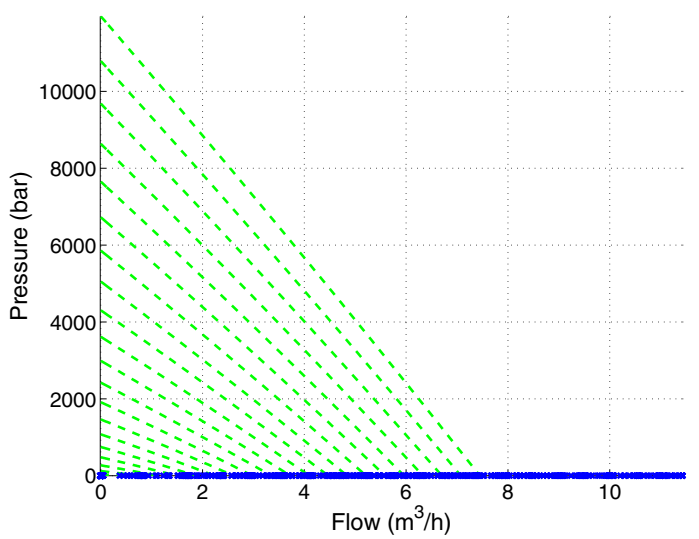

Figure 8: Best estimated pump-curves using hydraulic power with flow based on minimum Euclidean distance (base curve: solid red, estimated curves: dashed green, measurements: blue points).

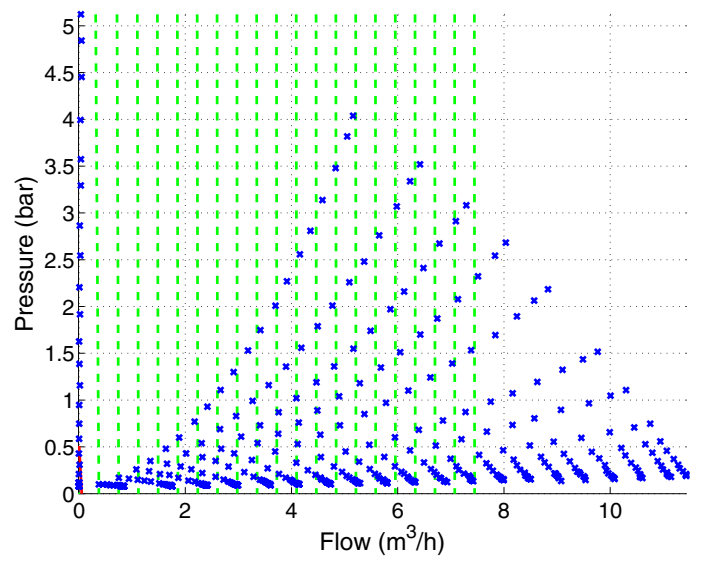

Figure 9: Zoomed version of the best estimated pump-curves using hydraulic power with flow based on minimum Euclidean distance (base curve: solid red, estimated curves: dashed green, measurements: blue points).

seen that the pump curves are almost vertical. The reason for this is unclear, but several factors is suspected of influencing this behavior. One factor is the Euclidean distance that is used to find the point on the pump-curve that is closest to any given measurement. By changing the shape of the curve, the point closest to the measurement can be moved considerably in both flow and pressure values. Another factor is the fact that it is the multiplication of the flow and the pressure that determines the hydraulic power. By varying these values correspondingly it is thus possible to obtain the same hydraulic power for a number of different pressure/flow combinations. It is, however, clear that the pump-curves obtained using this method are useless.

Another set of experiments was made for the case when $\tilde{Q}$ was determined from the measured flow $\hat{Q}$. The evolution of the best individual throughout the 30 runs is plotted in figure 10 .

The evolution again shows a clear tendency. This time, the $a_{0}$ parameter settles close to 0 in all runs, and the $b_{0}$

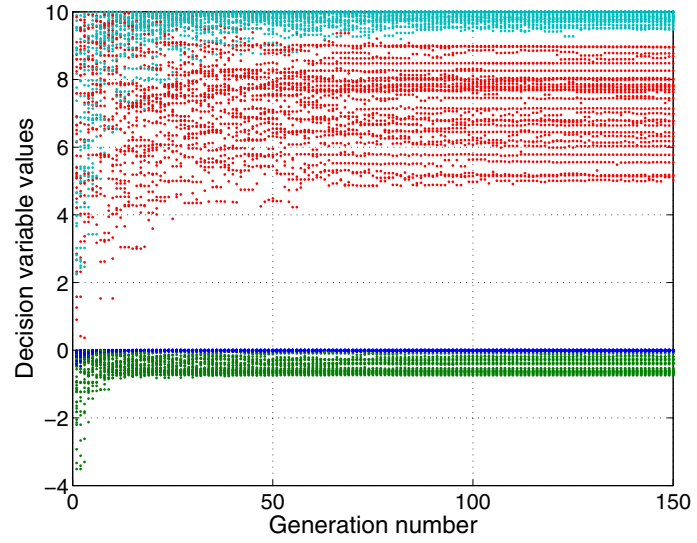

Figure 10: Distribution of pump-curve parameters during evolution using hydraulic power with measured flow ( $a_{0}$ : blue, $b_{0}$ : green, $c_{0}$ : red, $\omega_{0}$ : cyan).

parameter settles at values above -1 . It is also quite evident that the base speed $\omega_{0}$ settles at high speeds above 9. Again, the parameter $c_{0}$ does not converge to any specific value but has a tendency towards high values in the range [5,9].

The best set of parameters is obtained for the parameter values:

$\mathrm{a}_{0}=-0.00, \mathrm{~b}_{0}=-0.67, \mathrm{c}_{0}=8.27$, and $\omega_{0}=9.99$.

The best set of parameters is quite similar to the best parameters obtained through the various runs, so the obtained pump-curves should be characteristic for this method. The pump-curves obtained using the best set of parameters are plotted in figure 11.

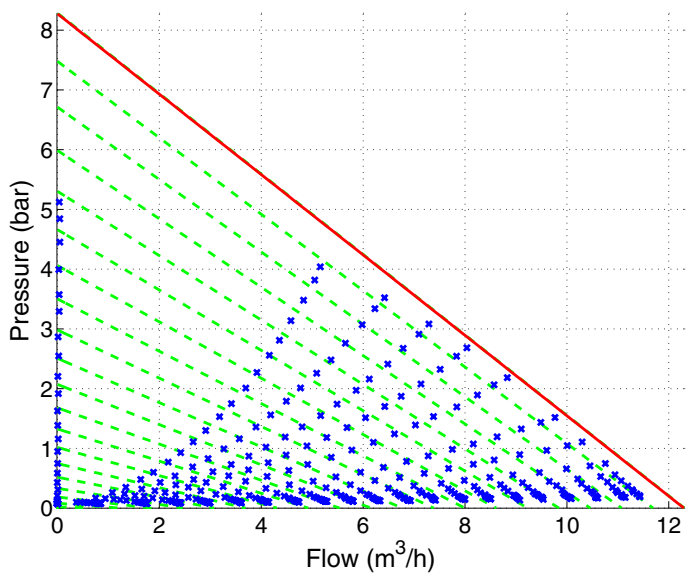

Figure 11: Best estimated pump-curves using hydraulic power and measured flow (base curve: solid red, estimated curves: dashed green, measurements: blue points).

As can be seen from the figure, the pump-curves are straight lines. Even though the pump-curves are quite good approximations when it comes to first order approximation of the measurements, they do not have the contribution from the second order term that would make them useful as pumpcurves. However, it should again be emphasized that the hydraulic power method has some flexibility in the multiplication of the pressure and flow and thus can exploit certain 
weaknesses in the problem. This is most likely what has caused these unusable pump-curves.

Using the proposed methods a lot of interesting results have been obtained. It is now time to compare them to each other in order to draw some conclusions.

\section{CONCLUSION}

This paper has presented some ways to obtain the best set of parameters for a general formulation of pump-curves based on affinity laws. The methods took different properties of the general formulation into account when estimating the parameter values.

Three different methods were proposed that were based on Euclidean distances, speed differences, and hydraulic power respectively. Each of the methods were capable of producing results, but the quality of the results varied considerably.

The method using hydraulic power was split into two parts, but both parts failed in producing results that could be considered adequate estimates. One of the parts produced curves that were outside acceptable ranges, and the other part only produced linear approximation that were inferior estimates of the pump-curves.

The remaining methods, using Euclidean distances and speed differences, both produced pump-curves that were of acceptable quality. This provides credibility to the approximated formulation using the affinity laws and indicates that they are of adequate precision for making design decisions regarding different pumps.

The pump-curves found using Euclidean distances mainly emphasized on higher base speeds $\omega_{0}$, which made them most applicable for high speeds. In order to obtain results for lower base speeds it would be necessary to reduce the range for the base speed before running the algorithm.

The method using speed differences produced different approximations for a variety of speeds in the different runs. For that method it might thus be necessary to run the algorithm a number of times in order to get a good approximation around a certain range, or the range of $\omega_{0}$ should be reduced to a desired range prior to estimation.

When looking at the measurements it can be seen that they are somewhat skewed towards higher flows. This is due to the way the measurements were made. This skewness of the measurements might have influenced the estimated parameters to some extent, and might even be the cause for the hydraulic power method to fail. However, it is interesting to notice that even though the measurements had this distribution it was still possible to obtain usable results using some of the methods.

In Section 4.1 it was mentioned that the power consumption was reduced at higher speeds. It was believed this was due to the control of the motor running the pump, as it reduced the speed when subjected to high loads. The method using Euclidean distances did make an effort to include the points of high load and might thus have been affected by this reduction of speeds at higher loads. The method using speed differences did not show the same sensitivity towards these high load measurements at very low flows. Some further investigations into this issue is recommended before relying too heavily on the obtained results.

Based on the results obtained the two best methods were the Euclidean distance based method and the speed difference method. Computationally, the speed difference method is preferable as it is less computational intensive. When it comes to precision, both methods seem reasonable even though the Euclidean based method seem to be limited to higher speeds and might be somewhat sensitive to high load conditions. It is thus the opinion of the authors that the preferred method for estimating the parameters should be the one based on speed differences. Refinement of the base speed, such that the precision in the desired operating range can be increased, can be done by restricting the possible range of $\omega_{0}$ before the estimation algorithm is executed.

\section{ACKNOWLEDGMENTS}

The authors would like to thank Mohammad Najat Anwar, Karsten B. Rasmussen, and Anh Tuan Kieu for their assistance in connection with modelling issues and performing experiments on the system.

\section{REFERENCES}

[1] Y.-H. Dai. New properties of a nonlinear conjugate gradient method. Numerische Mathematik, 89:83-98, 2001.

[2] Y.-H. Dai and L.-Z. Liao. New conjugacy conditions and related nonlinear conjugate gradient methods. Applied Mathematics and Optimization, 43:87-101, 2001.

[3] K. Deb. Multi-Objective Optimization using Evolutionary Algorithms. John Wiley \& Sons, Ltd., West Sussex, England, 1st edition, 2001.

[4] D. E. Goldberg. The Design of Innovation: Lessons from and for Competent Genetic Algorithms. Genetic Algorithms and Evolutionary Computation. Kluwer Academic Publishers, Norwell, MA, 2002.

[5] Grundfos A/S. Pump handbook. Website, 2007. http://net.grundfos.com/doc/webnet/ professional_profile/int/documentation.html.

[6] C. Kanzow, N. Yamashita, and M. Fukushima. Levenberg-marquardt methods for constrained nonlinear equations with strong local convergence properties. Journal of Computational and Applied Mathematics, 172:375-397, 2004.

[7] G. K. M. Pedersen and Z. Yang. Efficiency optimization of a multi-pump booster system. In Genetic and Evolutionary Computation GECCO-2008, Atlanta, GA, 2008. ACM Press.

[8] K. Sastry. Single and multiobjective genetic algorithm toolbox for matlab in c++. IlliGAL Report No. 2007017, University of Illinois at Urbana-Champaign, Illinois Genetic Algorithms Laboratory, Urbana, IL, 2007.

[9] T.-L. Yu, K. Sastry, D. E. Goldberg, and M. Pelikan. Population sizing for entropy-based model building in genetic algorithms. IlliGAL Report No. 2006020, University of Illinois at Urbana-Champaign, Illinois Genetic Algorithms Laboratory, Urbana, IL, 2006. 